\title{
Influence of Iron Oxide Particles on the Strength of Ball-Milled Iron
}

\author{
Donald R. Lesuer ${ }^{1}$, Chol K. Syn ${ }^{1}$ and Oleg D. Sherby ${ }^{2}$ \\ ${ }^{1}$ Lawrence Livermore National Laboratory, Livermore, CA 94551, USA \\ ${ }^{2}$ Department of Materials Science and Engineering, Stanford University, Stanford, CA 94305, USA
}

Detailed microstructural and mechanical property studies of ball-milled iron, in the powder and consolidated states, are reviewed and assessed. The analyses cover three and one-half orders of magnitude in grain size (from $6 \mathrm{~nm}$ to $20 \mu \mathrm{m}$ ) and focus on the influence of oxide particles on the strength. The study includes the early work of Jang and Koch, Kimura and Takaki and continues with the more recent work of Umemoto et al. and Belyakov, Sakai et al. It is shown that the major contributors to strength are the nano-oxide particles. These particles are created by adiabatic shear banding during ball-milling leading to a bimodal distribution of particles. The predicted strength from particles, $\sigma_{\mathrm{p}}$, is given by $\sigma_{\mathrm{p}}=B \cdot\left(D_{\mathrm{s}}{ }^{*}\right)^{-1 / 2}$ where $D_{\mathrm{s}}{ }^{*}$ is the surface-to-surface interparticle spacing, and $B=395 \mathrm{MPa} \cdot \mu \mathrm{m}^{1 / 2}$. A model is proposed that accounts for the influence of the bimodal particle size distribution on strength. [doi:10.2320/matertrans.47.1508]

(Received January 18, 2006; Accepted March 31, 2006; Published June 15, 2006)

Keywords: ball milled iron, strength, oxide particle strengthening, grain strengthening, nano-oxide, ultra-fine grains

\section{Introduction}

Many novel methods have been pursued to develop high strength iron-base materials with good ductility. New processes have centered on approaches involving severe plastic deformation in the ferrite temperature range. The objective is to create nano-ferrite grain sizes containing high angle grain boundaries. Ball-milling is an attractive approach to achieve such a goal. This is because severe plastic deformation is readily achieved. The high strain rate during ball-milling, typically $10^{4} \mathrm{~s}^{-1}$, imparts high flow stresses. The combination of large plastic deformation and high flow stress leads to the creation of fine dislocation cells, subgrains and grains. Grain sizes in the range of 6 to $100 \mathrm{~nm}$ have been observed in the ball-milled powders, ${ }^{1-5)}$ and as fine as 80 to $500 \mathrm{~nm}$ in the consolidated state. ${ }^{6-13)}$ Nominally pure iron powders are used as a starting material. The presence of iron oxide, as $\mathrm{Fe}_{3} \mathrm{O}_{4}$ particles, is evident after ball-milling and after consolidation. It is generally believed that the oxide particles do not contribute to the strength. It is considered, however, that the oxide particles contribute towards stabilizing the fine grain size and inhibit grain growth during annealing. The conclusion is that the grain size solely determines the strength and that the Hall-Petch relation is obeyed. The purpose of this paper is to investigate the possible influence of iron oxide particles, as an independent strengthening mechanism, on the mechanical properties of ball-milled iron. Data are analyzed ${ }^{1-13)}$ over the range of grain sizes from $6 \mathrm{~nm}$ to $20 \mu \mathrm{m}$ and volume fraction of oxide particles from trace amounts to 8 volume percent.

\section{Materials and Processes Evaluated in This Study}

The microstructure and resulting properties of ball-milled materials are dependent on many variables. Among these are the initial and final compositions of the iron-base material, the powder size, the composition and size of the milling balls, and the time/temperature history of ball milling and of consolidation. Table 1 is a summary of a number of investigations that are pertinent to the present objectives of interpreting the strength of ball-milled iron. Investigations are listed from 1990 to the present time. Ball milling time typically ranged from 20 to 500 hours. Initial composition of the powders ranged from commercially pure iron powder $(99.2 \% \mathrm{Fe})^{2,3)}$ to $99.9 \% \mathrm{Fe}^{4)}$ Powder sizes were from 5 to 100 microns, and in one instance high purity iron chips, one $\mathrm{mm}$ thick, were used. ${ }^{4}$ The ferrite grain size range studied was from $6 \mathrm{~nm}$ to $20 \mu \mathrm{m}$. Specific studies of the hardness, microstructural changes and contamination of the powders as a function of ball milling time were made ranging from a few hours to 500 hours. The oxygen content was generally given, and a column is devoted to this variable in Table 1 . All compositions are in mass $\%$ unless indicated otherwise. The oxygen content was noted to increase from $0.2 \mathrm{mass} \%$ to about 0.47 mass $\%$ oxygen after 200 hours of ball-milling. ${ }^{13)}$ Examples of the change in composition of the powders with milling time were noted by Sakai et al.; ${ }^{6}$ ) the chromium content increased from zero to about $0.4 \%$, and the carbon content from 0.02 to $0.07 \%$ after 100 hours.

\section{Results}

Figure 1 shows the yield strength-grain size data plotted as logarithm of the strength as a function of logarithm of the grain size. This method avoids the commitment to the minus one-half exponent that is made by the typical approach of plotting the linear function of yield strength $\left(\sigma_{\mathrm{y}}\right)$ as a function of the grain size term $(L)^{-1 / 2}$, according to the Hall-Petch relation $\sigma_{\mathrm{y}}=\sigma_{\mathrm{o}}+k_{\mathrm{y}} \cdot L^{-1 / 2}$ where $\sigma_{\mathrm{o}}$ and $k_{\mathrm{y}}$ are material constants. Hardness numbers were converted to yield strength by $H_{\mathrm{v}}=3 \sigma_{\mathrm{y}}$ for hardness values below $H_{\mathrm{v}}=6.5^{7)}$ and $H_{\mathrm{v}}=2.5 \cdot \sigma_{\mathrm{y}}$ for hardness values above $6.5 .{ }^{12)}$ The line at the bottom of Fig. 1 is the predicted Hall-Petch relation for data on interstitial-free iron and steel evaluated by Armstrong et al. ${ }^{14)}$ and Tsuji et al. ${ }^{15)}$ The data from these two studies are plotted as the yield strength minus the matrix strength $\left(\sigma_{\mathrm{o}}\right)$. The value of $\sigma_{\mathrm{o}}=28 \mathrm{MPa}$ is from Cracknell and Petch. ${ }^{16)}$ The interstitial-free iron data was obtained on cast material and thus avoided the oxides typically found in powderprocessed metals. As can be seen, the ball-milled iron data are not compatible with the Hall-Petch prediction and are located much above the interstitial-free iron line. It will be 
Table 1 Investigations of Ball-Milled Iron.

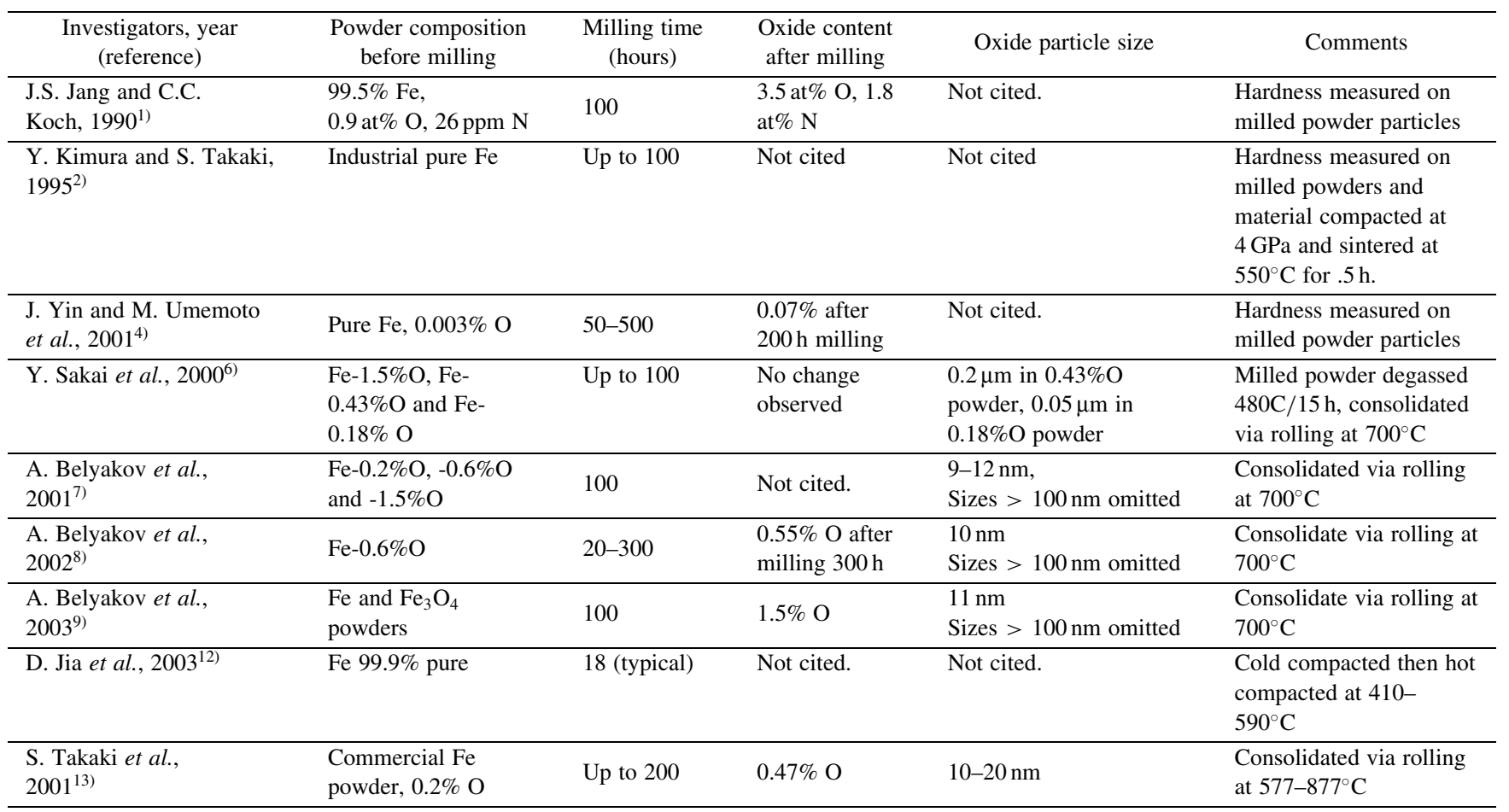

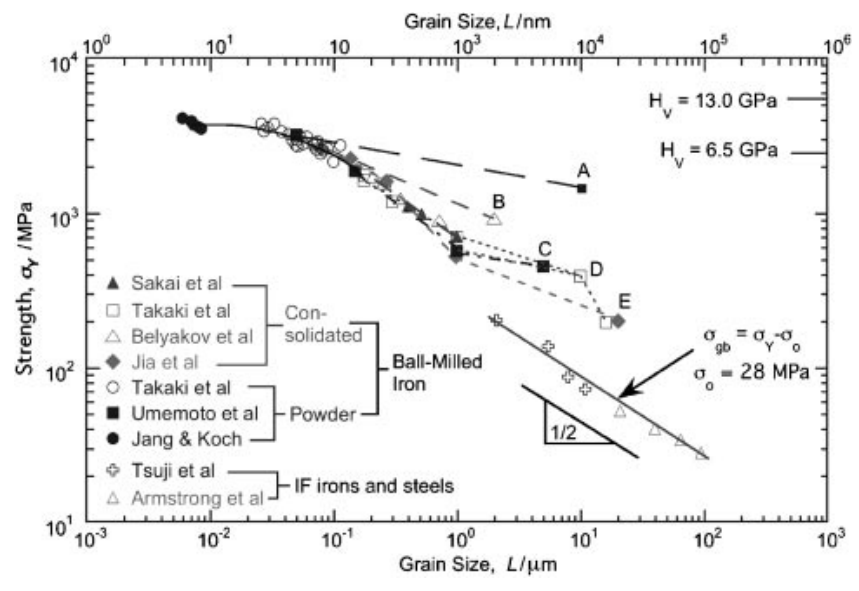

Fig. 1 Logarithm of the yield strength of ball-milled iron plotted as a function of the logarithm of the grain size. Also shown in the figure is data for wrought IF iron and steel. For these materials the strength is plotted as the yield strength minus the matrix strength $(28 \mathrm{MPa})$.

shown that the higher strength arises principally from oxide particle strengthening.

The data shown in Fig. 1 reveal three regions of dissimilar pattern. At ultra-fine grain sizes from $6 \mathrm{~nm}$ to about $60 \mathrm{~nm}$, the strength is seen to decrease gradually with increase in grain size. These data reveal the high hardness obtained in powders before consolidation. In the intermediate grain size range, from $0.12 \mu \mathrm{m}$ to about $1 \mu \mathrm{m}$, a steep dependence of strength with grain size is observed with a grain size exponent of about 0.7 rather than 0.5 . These data are mostly from consolidated powders. The temperature of consolidation varied widely, from as low as $400^{\circ} \mathrm{C}^{12)}$ to as high as $780^{\circ} \mathrm{C} .{ }^{13)}$ In the coarse grain size range, from about $1 \mu \mathrm{m}$ to $20 \mu \mathrm{m}$, the data are scattered with slopes drawn for individual studies that are generally less than 0.5 . These slopes are related to datum points given as A, B, C, D and E. These samples are associated with annealing treatments at high temperatures. Each slope was drawn by joining the individual datum points to the same processed material containing a finer grain size.

\section{Contribution of Grain Size and Particles to Strength}

The huge difference in strength between the ball-milled iron data and that for interstitial-free iron and steel (Fig. 1) is likely the result of oxide particle hardening. In order to establish the contribution of particles to strength, it is first necessary to establish the contribution of grain boundaries to strength. The answer lies in the Hall-Petch relation, and is given as:

$$
\sigma_{\mathrm{gb}}=\sigma_{\mathrm{y}}-\sigma_{\mathrm{o}}=k_{\mathrm{y}} \cdot L^{-1 / 2}
$$

where $\sigma_{\mathrm{gb}}$ is the strength contribution from grain boundaries, $\sigma_{\mathrm{Y}}$ is the experimentally determined yield strength, $\sigma_{\mathrm{o}}$ is the matrix strength, $L$ is the grain size and $k_{\mathrm{y}}$ is a material constant. The selection of an appropriate $\sigma_{0}, 28 \mathrm{MPa}$ for interstitial-free iron, ${ }^{16)}$ results in the expected Hall-Petch relation of minus one-half. This is in agreement with the dislocation pile-up theory for grain boundary strengthening. The best-fit value of $k_{\mathrm{y}}=260 \mathrm{MPa} \cdot \mu \mathrm{m}^{1 / 2}$ in eq. (1) can then be used to predict the grain boundary contribution to strength of iron for any grain size down to $2 \mu \mathrm{m}$.

The matrix strength, $\sigma_{\mathrm{o}}$, in iron, can be changed by various metallurgical factors such as solutes (interstitial and substitutional), particles, dislocation cells, and subgrains. These variables have been considered as additive and independent contributions ${ }^{17,18)}$ to the strength. In studies of Fe-C steels the present authors have found that particles dominate the 
strength of the iron matrix with the conclusion that other variables contribute comparatively little to the yield strength. ${ }^{19,20)}$ These investigations led to a prediction of the strength contribution from particles (that replaces $\sigma_{0}$ ) by the relation:

$$
\sigma_{\mathrm{p}}=A \cdot\left[D_{\mathrm{s}}{ }^{*}\right]^{-1 / 2}
$$

where $\sigma_{\mathrm{p}}$ is the strength increment arising from particle strengthening, and $D_{\mathrm{s}}{ }^{*}$ is the surface to surface inter-particle spacing. The constant A was estimated to be 320 $\mathrm{MPa} \cdot \mu \mathrm{m}^{1 / 2} \cdot{ }^{19,20)}$ The exponent for particle strengthening is the same as that for grain size strengthening and has been considered to have a dislocation pile-up origin. ${ }^{21)}$ Equation (3) describes the contribution of grain size and particles to the yield strength through eqs. (1) and (2).

$$
\sigma_{\mathrm{y}}=k_{\mathrm{y}} \cdot L^{-1 / 2}+A \cdot\left[D_{\mathrm{s}}{ }^{*}\right]^{-1 / 2}
$$

For investigations where particle size and volume fraction of particles were available, the value of $D_{\mathrm{s}}{ }^{*}$ was calculated from the following:

$$
D_{\mathrm{s}}^{*}=(3 / 2)^{0.5} \cdot\left\{\left[\pi /\left(4 \cdot f_{\mathrm{v}}\right)\right]^{0.5}-1\right\} \cdot d
$$

where $f_{\mathrm{v}}$ is the volume fraction of particles and $\mathrm{d}$ is the particle diameter.

There is a problem, however, with the application of eq. (3) to predicting the yield strength at fine grain sizes. This problem arises because experimental evidence on the strength of Fe-C steels indicates that grain size strengthening is less effective with decreasing grain size than predicted by the Hall-Petch relation beginning at about $2 \mu \mathrm{m}$.

\subsection{Breakdown of Hall-Petch relation in Fe-C steels.}

Since the publication of the Syn et al. paper, ${ }^{19)}$ extensive research and development studies on Fe-C steels have been pursued with the objective of achieving fine grain sizes. In general, the processing approaches used to develop the fine grain sizes involved various forms of severe plastic deformation. These studies were pursued through processing of wrought materials and of powder metallurgy materials.

Figure 2 shows a log-log plot of the yield strength of Fe-C steels as a function of the reported grain size. The three lowest strength materials (aged mild steel ${ }^{22}$ and two interstitial-free irons $)^{14,15)}$ are given as yield strength minus a matrix strength $(28 \mathrm{MPa})$. The slopes of the lines for this data equal minus one-half confirming the Hall-Petch relation. These investigations covered the grain size range from 2 to $100 \mu \mathrm{m}$. The two sets of data, however, differ in strength by a factor of three. No discussion or explanation has been given for this large ratio in strengths. It is undoubtedly related to the strengthening effect of iron carbide particles in the aged mild steel. Microstructures of the aged steel were not shown by Hall in his classic work on the aged steel. Nevertheless it is possible to make an estimate from eqs. (1) and (2). This is because the grain size is related to the interparticle spacing as a result of the Zener pinning mechanism during grain growth. ${ }^{23)}$ For spheroidized ultrahigh carbon steels, the relation between interparticle spacing and grain size was found to be $D_{\mathrm{s}}{ }^{*}=0.4 \cdot L .^{24)}$ The details of this relationship are described in the Appendix. Using this relation, one can determine the ratio of strengths of the two materials (aged

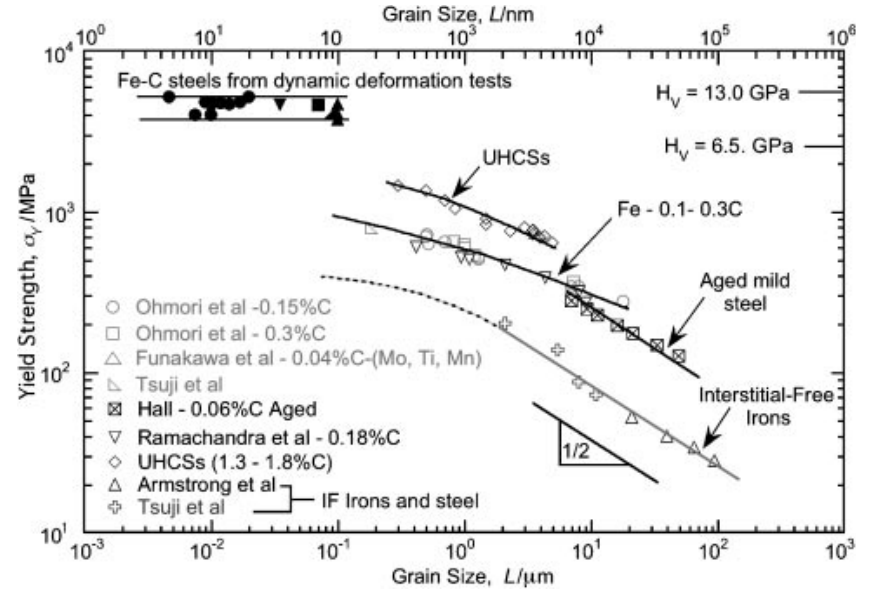

Fig. 2 Logarithm of the yield strength of iron-carbon steels plotted as a function of the logarithm of the grain size. Also shown in the figure is data for aged mild steel and two IF irons and steel. For these materials the strength is plotted as the yield strength minus the matrix strength (28 MPa).

mild steel and interstial-free iron) from eqs. (1) and (2). The ratio is equal to $\left(\sigma_{\mathrm{gb}}+\sigma_{\mathrm{p}}\right) / \sigma_{\mathrm{gb}}=2.94$, and this is in agreement with the factor of about three observed experimentally.

The remainder of the data shown in Fig. 2 does not agree with the Hall-Petch relation. The most serious discrepancy is seen with the data documented in the upper left section in Fig. 2 (labeled as steels from dynamic deformation tests). ${ }^{20)}$ These data are for Fe-C steels containing 0.6 to $1.3 \% \mathrm{C}$. The ultra-fine grain size data $(0.005$ to $0.1 \mu \mathrm{m})$ are seen to have the same high hardness indicating that the Hall-Petch relation is not applicable for $\mathrm{Fe}-\mathrm{C}$ steels at these ultra-fine grain sizes. These high hardness values were achieved by ball-milling (identified as solid circles in the figure), by ball-drop tests (identified by solid triangles) and by dynamic impact tests (identified as solid squares). ${ }^{20)}$ The other data in Fig. 2 are for the ultra-high carbon steels (UHCSs) ${ }^{19)}$ and for low carbon $(0.1$ to $0.3 \% \mathrm{C})$ steels $^{25-29)}$ at small grain sizes $(<2 \mu \mathrm{m})$. These data show slopes that are considerably smaller than the minus one-half exponent predicted from the Hall-Petch relation. We attribute the breakdown of the Hall-Petch relation for grain sizes less than $2 \mu \mathrm{m}$ to the decreasing effectiveness of dislocation pile-up at grain boundaries. Grain boundary migration and/or grain boundary sliding can occur more readily as the grain size is reduced leading to grain boundary relaxation. Takaki, Hidaka et al. ${ }^{13,30)}$ have suggested a similar proposal where stress accommodation mechanisms at grain boundaries occur that make grain boundaries less effective barriers to plastic flow. A dotted line is shown in Fig. 2 to indicate the predicted diminishing effect of grain size on the strength of pure iron at fine grain sizes. It is this curve that will be used to assess the contribution of particles to the strength of $\mathrm{Fe}-\mathrm{C}$ and $\mathrm{Fe}-\mathrm{O}$ materials by the following relation:

$$
\sigma_{\mathrm{p}}=\sigma_{\mathrm{y}}-\sigma_{\mathrm{gb}}=B \cdot\left[D_{\mathrm{s}}{ }^{*}\right]^{-1 / 2}
$$

The objective is to determine the value of $B$ by plotting $\sigma_{\mathrm{p}}$ as a function of $D_{\mathrm{s}}{ }^{*}$ with the inclusion of the many new data 


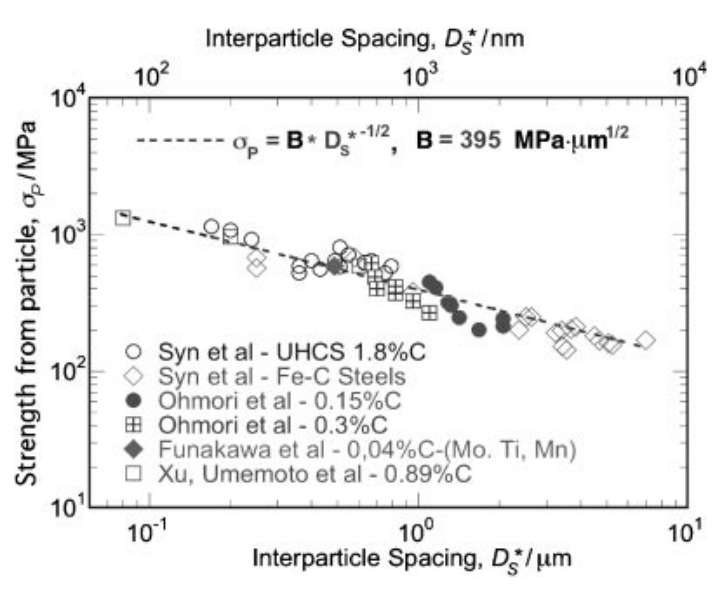

Fig. 3 The strength of iron-carbon steels from particles, $\sigma_{\mathrm{p}}$, plotted as a function of inter-particle spacing, $D_{\mathrm{s}}{ }^{*}$.

now available to those previously obtained for spheroidized low ${ }^{25-29)}$ and high carbon steels. ${ }^{19)}$

Figure 3 shows the logarithm of the strength from carbide particles, $\sigma_{\mathrm{p}}$, plotted as a function of the logarithm of $D_{\mathrm{s}}{ }^{*} \cdot{ }^{19,25,29,31,32)}$ The value of $D_{\mathrm{s}}{ }^{*}$ was calculated from eq. (4). A least squares fit of all the data plotted yielded an exponent of minus 0.56. This value of the exponent is close to the exponent of -0.5 proposed by Syn et al. ${ }^{19)}$ The pre-constant value of $B$ [in eq. (5)] is $395 \mathrm{MPa} \cdot \mu \mathrm{m}^{1 / 2}$. The value is higher than the pre-constant of $320 \mathrm{MPa} \cdot \mu \mathrm{m}^{1 / 2}$ calculated previously with less extensive data and with a different correction for the strength expected from grain size.

\subsection{Particle strengthening in iron-oxygen materials}

The particle strengthening relation developed for $\mathrm{Fe}-\mathrm{C}$ materials [eq. (5) with $B=395 \mathrm{MPa} \cdot \mu \mathrm{m}^{1 / 2}$ ] should be equally applicable to the iron-oxygen materials described in Fig. 1. The oxide particle strength, $\sigma_{\mathrm{P}}$, was calculated by subtracting the grain size strength, $\sigma_{\mathrm{gb}}$, from the yield strength. The corresponding values for inter-particle spacing, $D_{\mathrm{s}}{ }^{*}$, could not be determined by the use of eq. (4). This is because precise data on the size, distribution and volume fraction of the oxide particles were not available in most studies. Instead, it was decided to calculate the values of $D_{\mathrm{s}}{ }^{*}$ through the phenomenological relation $D_{\mathrm{s}}{ }^{*}=0.4 \cdot L$. Another constraint in analyses of the results relates to the influence of ball-milling time on the microstructure and strength. For example, only subgrains and cells are created in the initial stages of milling (e.g. 20 hours). These structures are retained even after consolidation. ${ }^{8)}$ The presence of subgrains and cells would not necessarily follow the same relation observed for grain/particle interactions. It was therefore decided to analyze data from ball-milling times of 50 hours or greater. As a result, data from Jia et al. were not analyzed since only 18 hours of ball-milling was used and subgrain boundaries can be expected to dominate the microstructure. These data, however, have been analyzed by considering the influence of subgrains and particles on the strength of ball milled iron. ${ }^{33)}$ Subgrain boundary strengthening is known to follow a different power relation than that observed for grain boundary strengthening. ${ }^{34)}$

Figure 4 shows the resulting correlation between $\sigma_{\mathrm{p}}$ and

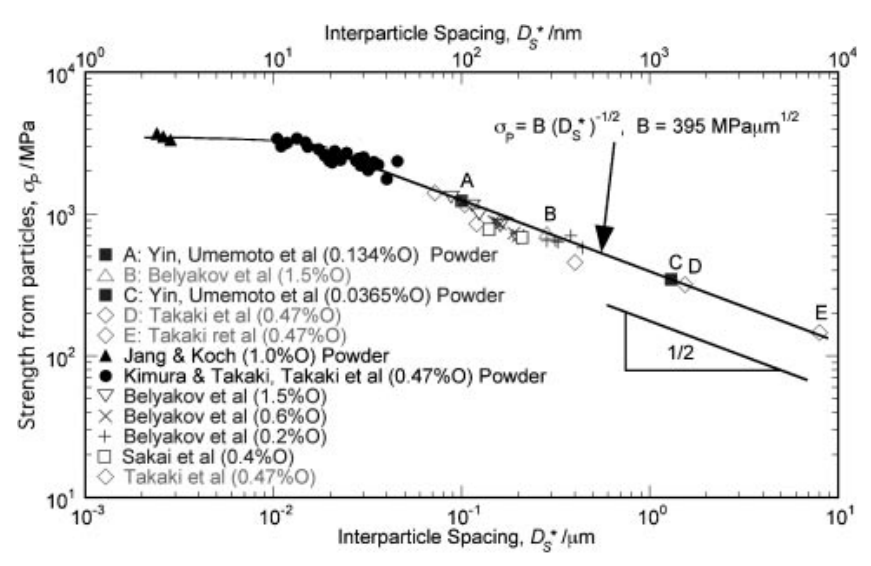

Fig. 4 Strength of ball milled iron-oxygen alloys from particles, $\sigma_{\mathrm{p}}$, as a function of inter-particle spacing, $D_{\mathrm{s}}{ }^{*}$. Values of $D_{\mathrm{s}}{ }^{*}$ were calculated from $D_{\mathrm{s}}{ }^{*}=0.4 \cdot L$, except for Points A to $\mathrm{E}$ as described in the text.

$D_{\mathrm{s}}{ }^{*}$. Most of the data agree with the predicted relation derived for the particle strengthening in $\mathrm{Fe}-\mathrm{C}$ steels. Data from investigations where $f_{\mathrm{v}}$ and $\mathrm{d}$ were documented ${ }^{7-9,11)}$ will be described in the discussion section. The Fe-O data shown in Fig. 4 extend the particle strengthening effect by a factor of four in inter-particle spacing (to $D_{\mathrm{s}}{ }^{*}=0.02 \mu \mathrm{m}$ ) relative to the data for the $\mathrm{Fe}-\mathrm{C}$ steels as recorded in Fig. 3. The particle strengthening effect, however, appears to diminish below about $0.02 \mu \mathrm{m}$. A possible explanation is that grain size refinement occurs more rapidly than particle refinement during ball-milling to achieve the highest strengths.

It is appropriate to examine the coarse grain size data, Points A, B, C, D, and E, shown originally in Fig. 1 and again in Fig. 4. This is because particle strengthening is the only plausible explanation for understanding the high strength of these materials. In Fig. 4, these five points are shown as predicted by the particle strengthening relation given by eq. (5). The values of $\left(D_{\mathrm{s}}{ }^{*}\right)$ represent the predicted interparticle spacings that exist within each of the coarse grain size materials. These predictions become justified when the particle size and volume fraction are known and the values of $D_{\mathrm{s}}{ }^{*}$ are confirmed from the application of eq. (4). Detailed microstructural evidence is not available for such calculations. Data points A and C from Yin, Umemoto et al., ${ }^{4)}$ however, give supportive evidence for particle strengthening. Both points are related to ball-milling followed by annealing at $800^{\circ} \mathrm{C}$. Point $\mathrm{A}$, with $\sigma_{\mathrm{p}}=1160 \mathrm{MPa}$, was obtained by ball-milling the iron powders for 500 hours and Point $\mathrm{C}$, with $\sigma_{\mathrm{p}}=346 \mathrm{MPa}$, was obtained by ball-milling for 100 hours. The authors worked with $99.9 \%$ pure iron containing an initial oxygen content of $0.003 \%$. The oxygen content was noted to increase from $0.003 \%$ to 0.07 mass $\%$ after 200 hours of ball-milling. A linear interpolation to 100 hours predicts 0.0365 mass $\% \mathrm{O}\left(f_{\mathrm{v}}=0.00195\right)$, and a linear extrapolation to 500 hours predicts an oxygen content of 0.134 mass $\%$ $\left(f_{\mathrm{v}}=0.0072\right)$. An estimate of the particle size is available from the work of Belyakov et al. ${ }^{9)}$ who showed $d=13 \mathrm{~nm}$ in ball-milled, consolidated and annealed Fe-O materials.

These values of $f_{\mathrm{v}}$ and d allow for calculation of $D_{\mathrm{s}}{ }^{*}$ with eq. (4) and then, $\sigma_{\mathrm{P}}$ is readily calculated with eq. (5). The calculated results for the 500 hour ball-milled iron (Point A) 
give a predicted strength of $1020 \mathrm{MPa}$. This strength is in close agreement with the experimental strength from particles of $1160 \mathrm{MPa}$ giving good support to eq. (5). A similar calculation for Point $\mathrm{C}$ gave a predicted strength of $718 \mathrm{MPa}$. This value is much higher than the experimental value of $346 \mathrm{MPa}$. The discrepancy is related to the fact that after 100 hours of ball-milling only a fraction of the oxide particles will be in the nano-size range. The remaining oxide exists as coarse particles in work-hardened regions. The details of particle size distribution with ball-milling time, and the influence of the resulting bi-modal distribution on strength, are covered in the discussion section.

\section{Discussion}

The early work of Jang and Koch ${ }^{1)}$ and of Takaki, Kimura et al. ${ }^{2,3,13)}$ have shown the uniqueness of ball-milling iron powders as a means of developing new ultra-high strength iron-base materials. Umemoto and colleagues ${ }^{4,5)}$ have thoroughly evaluated the unusual, and unexpected, changes in the structure and hardness of iron powders as a function of ballmilling time and subsequent annealing. Belyakov, Sakai, Tsuzaki and their colleagues ${ }^{6-11)}$ have evaluated the microstructure of ball-milled iron and iron-oxide powders after ball-milling and after consolidation. Thermal mechanical processing of powders for consolidation, and subsequent annealing are variables that influence microstructure-property relations. These extensive studies are now discussed with respect to the influence of iron oxide particles on the strength of ball-milled iron and iron alloys.

\subsection{Work-hardened and transformed states during ball- milling}

The change in microstructure with ball-milling time of iron powders has been investigated. ${ }^{1-6)}$ The initial stage of ballmilling shows the creation of dislocation cells and subgrains. This is the work-hardening stage and the hardness of the powders increases with ball-milling time. ${ }^{2-4,6,13)}$ As the ballmilling time is further increased, Yin, Umemoto and their colleagues $^{4)}$ have shown that a new structure appears that adjoins the work-hardened region in each powder. The sharp boundary between the two structures indicates a sudden transformation process. The transformation creates a discontinuity in the hardness; the transformed material is typically 2 to 3 times higher in strength than the workhardened material. Figure 5 illustrates the discontinuities in a plot of the Vickers hardness of the $99.9 \%$ iron powders as a function of ball-milling time. Two distinct hardness plateaus are observed. A forbidden zone of hardness exists. It is proposed that the transformed structure involves the creation of nano-ferrite grains and nano-oxide particles. This structure was first recorded at about 50 hours of ball-milling and became the only structure observed at 500 hours. Most microstructure investigations of ball-milled iron were evaluated at 100 hours of ball-milling time. It is proposed that approximately equal amounts of each microstructural state co-exist at 100 hours.

Figure 5 shows that the hardness ratio of the two structures is about 2.5 in the region where they co-exist. Remarkably, this ratio stays about the same even after annealing the ball-

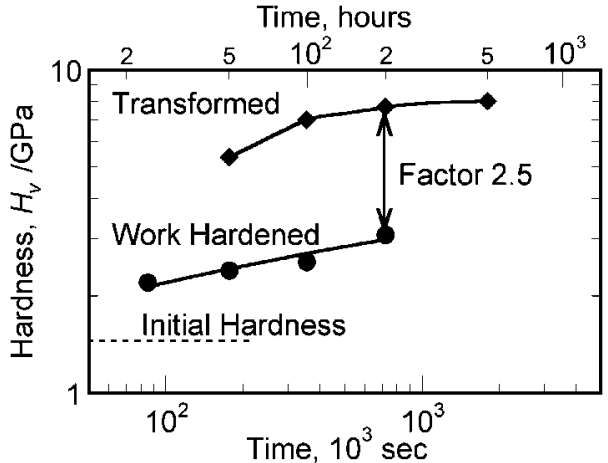

Fig. 5 The Vickers hardness of pure iron as a function of ball-milling time, after Yin, Umemoto, Liu and Tsuchiya.

milled powders above the alpha-gamma transformation temperature. ${ }^{4)}$ The $H_{\mathrm{v}}$ hardness was $1.3 \mathrm{GPa}$ for the transformed material and $0.6 \mathrm{GPa}$ for the work-hardened material after annealing at $1000^{\circ} \mathrm{C}$. These dissimilar strengths, with a ratio of 2.2 , cannot be explained by grain size differences since the alpha-gamma transformation should have eliminated any differences in grain size that may have existed. Umemoto et al. showed similar discontinuities in structure and hardness for ball-milled $\mathrm{Fe}-\mathrm{C}$ powders. ${ }^{31,32)}$

Mechanisms of transformation to nano-structures in ballmilled iron and $\mathrm{Fe}-\mathrm{C}$ powders have been considered by Umemoto. ${ }^{5)}$ He concluded that "the real mechanism is not clear". The present authors have developed a mechanism based on adiabatic shear banding. It is proposed that shear banding will take place when sufficient strain energy is reached in the work-hardened material for the case of ballmilling Fe-C steels. ${ }^{20,35,36)}$ The large strain induced in the shear band will refine the carbides and heat the material, adiabatically, to cause transformation from ferrite to austenite. Re-transformation is essentially athermal since rapid cooling occurs and the final structure consists of nanocarbides and ultra-fine ferrite grains. The end result is a very hard material in the order of $H_{\mathrm{v}}=11$ and higher.

It is proposed that the same mechanism is applicable to ball milling of pure iron powders. The difference is that one must use the $\mathrm{Fe}-\mathrm{O}$ phase diagram ${ }^{37)}$ for interpretation instead of the $\mathrm{Fe}-\mathrm{C}$ diagram. Oxygen is soluble in ferrite, 0.031 mass $\%$ at the transformation temperature, $910^{\circ} \mathrm{C}$, and virtually insoluble in gamma $(<0.002$ mass $\%)$. Therefore, during adiabatic shear banding, the small amount of oxygen in solution in the ferrite will precipitate as iron oxide upon transformation to gamma iron. The rapid cooling during re-transformation will not allow sufficient time to dissolve the precipitated particles. It is proposed that these precipitates are the predecessors to the nano-size particles observed by Belyakov et al. ${ }^{8,9,11)}$ in the consolidated state. The work-hardened regions will not show the nano-size particles since no transformation takes place.

\subsection{Microstructure-property relations in Fe-O materi- als}

The complexity of assessing the microstructural changes on the properties of ball-milled iron containing oxygen is evident from the work of Belyakov, Sakai, et al. ${ }^{6-9)}$ A major 


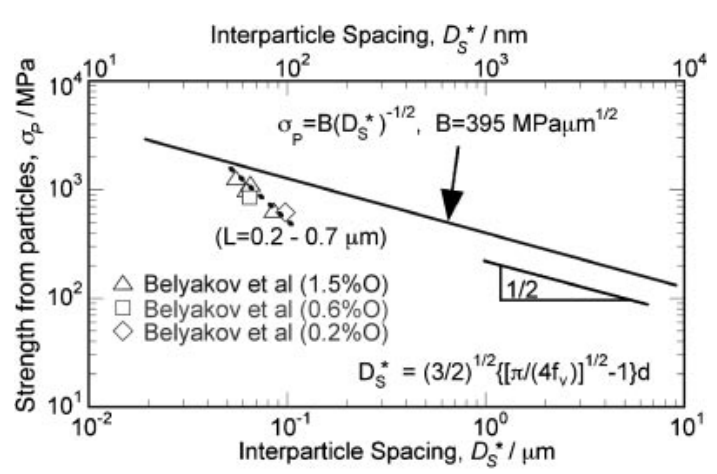

Fig. 6 Strength of ball milled iron-oxygen alloys, $\sigma_{\mathrm{p}}$, as a function of interparticle spacing, $D_{\mathrm{s}}{ }^{*}$. Values of $D_{\mathrm{s}}{ }^{*}$ were calculated from $D_{\mathrm{s}}{ }^{*}=$ $1.22 \cdot\left\{\left[\pi /\left(4 \cdot f_{\mathrm{v}}\right)\right]^{0.5}-1\right\} \cdot d$.

purpose of these studies was to stabilize the fine grain size by oxide particle pinning. The authors documented the size of oxide particles and grains as a function of oxygen content, ball-milling time, consolidation and subsequent annealing. Mechanical properties were evaluated principally with the consolidated material. Their major emphasis was on the influence of grain size on the yield strength of the $\mathrm{Fe}-\mathrm{O}$ powders containing $0.2,0.4,0.6$ and 1.5 mass $\% O$. The corresponding total volume fractions of oxide are 0.0107 , $0.0213,0.032$ and 0.08 . The oxide was introduced by controlled oxidation of the powders. Ball-milling broke up the oxide skin to form particles that were continually refined during the ball-milling process. ${ }^{6)}$ Consolidation was by canning the powders into a steel pipe and warm rolling extensively to a true strain of 2.0 at $700^{\circ} \mathrm{C}$. The authors recorded the presence of nano-size oxide particles in the transformed regions of the consolidated materials. They calculated the size and volume fraction of nano-size particles in the case of three oxide content materials (namely 0.2, 0.6 and 1.5 mass $\%$ oxygen). The respective particle sizes were $9 \mathrm{~nm}, 10 \mathrm{~nm}$ and $11 \mathrm{~nm}$, and respective volume fractions were $0.008,0.02$ and 0.03 . Particle sizes greater than $50 \mathrm{~nm}$ were not quantified for size and distribution. The coarser sizes are associated with the work-hardened region described by Yin, Umemoto and their colleagues. ${ }^{4)}$ Long time annealing experiments were conducted to observe the growth of particles and grains for the 1.5 mass $\%$ O material. ${ }^{9)}$

It is possible to calculate $D_{\mathrm{s}}{ }^{*}$ from the authors' data on $f_{\mathrm{v}}$ and nano-particle size $d$ by means of eq. (4) for the $0.2,0.6$ and 1.5 mass $\% \mathrm{O}$ materials in the consolidated state after ball-milling for 100 hours. ${ }^{9,10)}$ Figure 6 shows the predicted particle strengths as a function of $D_{\mathrm{s}}{ }^{*}$ from the microstructure studies and compares these data to the straight line prediction for particle strengthening from eq. (5). All three Fe-O materials show high strengths from the presence of particles that confirms our proposal that particles contribute to the strength of ball-milled iron. The strengths are not as high as expected from the predicted line. Furthermore, the extensive data for the 1.5 mass $\%$ O material shows a steeper slope than predicted by the $\left(D_{\mathrm{s}}^{*}\right)^{-1 / 2}$ relation. In contrast to this result, the calculation of $D_{\mathrm{s}}{ }^{*}$ by $D_{\mathrm{s}}{ }^{*}=0.4 \cdot L$ gives a closer agreement with the predicted strength as given by the triangle points in Fig. 4. A possible explanation lies in the

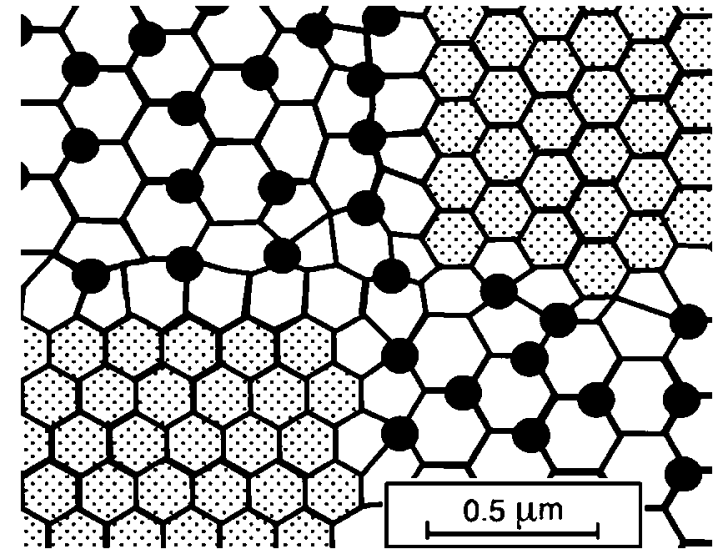

Fig. 7 Proposed bi-modal model illustrating the distribution of particles in work-hardened and transformed states in consolidated ball milled iron$1.5 \%$ oxygen.

complex changes in oxide particle morphology with processing.

Sakai et al. have shown that the hardness of ball-milled Fe$\mathrm{O}$ materials is a function of oxygen content and ball-milling time. ${ }^{6)}$ The highest hardness achieved was $H_{\mathrm{v}}=10.2$ for the $1.5 \% \mathrm{O}$ material. Upon consolidation the hardness decreased by a factor of two, to $H_{\mathrm{V}}=5.2$. The same ratio for hardness decrease is observed for the 0.2 and 0.4 mass $\% \mathrm{O}$. These decreases in hardness can be attributed to nano-particle growth during warm working at $700^{\circ} \mathrm{C}$ to the size of about $10 \mathrm{~nm}$ documented by Belyakov et al. for the consolidated materials. An estimate of the predicted particle size from ball milling prior to consolidation is readily made from eqs. (4) and (5). The hardness ratio is proportional to $d^{-1 / 2}$ indicating a four-fold decrease in particle size. Therefore, it is predicted that a particle size of $2.5 \mathrm{~nm}$ is present in the ball-milled powders. This small size is consistent with the predicted size of carbides of $1.6 \mathrm{~nm}$ in shear band studies in ultrahigh carbon steel. ${ }^{20)}$ Only limited studies have been made on the size of oxide particles that are greater than $50 \mathrm{~nm}$. In the case of the 0.2 mass $\% \mathrm{O}$ material, ball-milling for 100 hours reduced the initial oxide particle size of $0.3 \mu \mathrm{m}$ to $50 \mathrm{~nm} .{ }^{6)} \mathrm{In}$ the case of the 1.5 mass $\%$ O material, ball-milling for 50 hours reduced oxide particles from 100 to $300 \mathrm{~nm}$ [ref. 6, Fig. 4(f)]. We project a reduction of the particle size, after 100 hours of ball-milling, from 50 to $150 \mathrm{~nm}$. These are the sizes predicted in the work-hardened region. It would seem that a rigorous analysis of the data shown in Fig. 6 based on the work of Belyakov et al. requires a model that considers a bi-modal distribution of oxide particles.

Figure 7 illustrates a proposed model involving a distribution of two different regions of oxide particles in the 1.5 mass \% O material after ball-milling for 100 hours followed by consolidation. One region shows $100 \mathrm{~nm}$ oxide particles (the work-hardened region) that are attached to grains that are in the order of $0.25 \mu \mathrm{m}$ in size. This region follows the pattern expected from Zener pinning. The homogeneous refinement of particles and grains is a result of the large strain during extensive warm rolling of the compacted powders. This second region shows the $10 \mathrm{~nm}$ oxide particles (the transformed region) contained within 
grains that are in the order of $0.2 \mu \mathrm{m}$ in size. The nano-oxide particles are shown to be contained within the fine grains (Ref. 11), Fig. 2). In this case, it would appear that Zener pinning is not operative during the warm rolling process for consolidation. This observation is consistent with the proposed microstructure at the end of ball-milling prior to consolidation. This microstructure consists of ultra-fine oxide particles $2.5 \mathrm{~nm}$ in size adjoining similar size grains. That is, the changes in microstructure during ball-milling are controlled by the Zener pinning processes and the relation $D_{\mathrm{s}}{ }^{*}=$ $0.4 \cdot L$ is obeyed. The subsequent consolidation process, however, involves extensive warm rolling deformation, and this provides sufficient driving force for the nano-size grains to overcome the Zener pinning force. As a result the final grain size in the transformed region approaches the size observed in the work-hardened region.

The bi-modal distribution of particles shown in Fig. 7 can be used to calculate the strength contribution from particles for the consolidated 1.5 mass $\% \mathrm{O}$ material. According to Belyakov et al., the nano-oxide particles take up to 3 vol $\%$ of oxide and the remaining particles take up 5 vol\% of oxide. The method for determining the volume fraction of nano-size particles was to count the number of particles in a given region where they were observed by TEM. The strength from the nano-oxide particles from eqs. (4) and (5) is $\sigma_{\mathrm{p}}=$ $1762 \mathrm{MPa}$ (with $f_{\mathrm{v}}=0.03$ and $d=10 \mathrm{~nm}$ ). The strength from the work-hardened region, with $f_{\mathrm{v}}=0.05$ and $d=$ $100 \mathrm{~nm}$, is $\sigma_{\mathrm{p}}=655 \mathrm{MPa}$. These two strengths represent the hard material and the soft material respectively. These values are to be compared with $\sigma_{\mathrm{p}}=1418 \mathrm{MPa}$ calculated from the experimentally determined yield strength $(1768 \mathrm{MPa})$ and grain size $(0.2 \mu \mathrm{m})$ from Sakai et al. $\left.{ }^{6}\right)$ The complexity of the distribution of particles makes for some uncertainty as to how the two regions shown in Fig. 7 might contribute to the total strength. It was decided to calculate the strength from the bi-modal distribution by assigning a fractional contribution from each region according to the following additive relation:

$$
\sigma_{\mathrm{p}}(1418 \mathrm{MPa})=G_{1}(1762 \mathrm{MPa})+G_{2}(655 \mathrm{MPa})
$$

where $G_{1}$ is the effective volume fraction of the nano-oxide particle region and $G_{2}$ is the effective volume fraction of the work-hardened region. The relation given by eq. (6) predicts $G_{1}=0.3$ for the nano-size region and $G_{2}=0.7$ for the workhardened region. We interpret this to mean that $30 \%$ of nanooxide particles contribute to the strength with the remaining $70 \%$ coming from the $100 \mathrm{~nm}$ size particles.

The steep slope of the $\sigma_{\mathrm{p}}-D_{\mathrm{s}}{ }^{*}$ relation for the 1.5 mass $\%$ oxygen material, shown in Fig. 6, can be explained by the changes in the region around the nano-oxide particles with annealing. Particle depleted zones were observed to form during annealing as a result of the dissolution of nano-size particles by the migrating grain boundaries. ${ }^{9)}$ The depleted zones will reduce the value of $G_{1}$ in eq. (6). A value of $G_{1}=0.125$ is calculated for the lowest strength consolidated material annealed at $900^{\circ} \mathrm{C}$ (Fig. 6). In this case, $\sigma_{\mathrm{p}}=$ $750 \mathrm{MPa}$ and $\sigma_{\text {nano }}=1490 \mathrm{MPa}$ assuming $d=14 \mathrm{~nm}$. These calculations indicate that the coarse iron oxide particles, i.e. $100 \mathrm{~nm}$ in size, are the major contributors to the strength of the consolidated material annealed at $900^{\circ} \mathrm{C}$.
The bi-modal description of the structure of ball-milled and consolidated $\mathrm{Fe}-\mathrm{O}$ materials is realistic but is quantitatively limited. This is because the values of the fractional contribution, $G_{1}$ and $G_{2}$ are simply numbers to obtain a good fit of the proposed model. There is no proof of their validity with current microstructural studies. As a result, it is likely more appropriate to assess the contribution to strength from particles with the phenomenological relation between interparticle spacing and grain size by $D_{\mathrm{s}}{ }^{*}=0.4 \cdot L$ in the fine grain size range. It appears to represent a more global overview of the grain-size/interparticle-spacing relation that describes the strength.

\subsection{Relation for describing the yield strength of particle strengthened materials}

The analyses made in the present paper on the strength of ball-milled iron have separated the influence of particles and grain size on the yield strength. In the medium to coarse grain size range ( 2 to $100 \mu \mathrm{m}$ ), the yield strength is described by the following relation:

$$
\sigma_{\mathrm{y}}=260 \cdot L^{-1 / 2}+395 \cdot\left[D_{\mathrm{s}}{ }^{*}\right]^{-1 / 2}
$$

where the numerical constants are in units of $\mathrm{MPa} \cdot \mu \mathrm{m}^{1 / 2}$. Under the condition that $D_{\mathrm{s}}{ }^{*}=0.4 \cdot L$ (or $L=2.5 \cdot D_{\mathrm{s}}{ }^{*}$ ), eq. (7) can be rewritten in terms of $D_{\mathrm{s}}{ }^{*}$ or $L$, as follows:

$$
\sigma_{\mathrm{y}}=560 \cdot\left[D_{\mathrm{s}}{ }^{*}\right]^{-1 / 2} \quad \text { or } \quad \sigma_{\mathrm{y}}=885 \cdot L^{-1 / 2}
$$

These relations could be called dispersion-strengthened Hall-Petch equations. The cooperative aspect of particle and grain size strengthening in iron-carbon and iron-oxygen steels was suggested by Sherby et al. ${ }^{24)}$ and by Belyakov et al. ${ }^{11)}$

At grain sizes finer than about $2 \mu \mathrm{m}$, the influence of grain size on the yield strength decreases because grain boundary sliding makes the boundary a less effective barrier to slip. ${ }^{20)}$ It is in this grain size range that particles can dominate the contribution to the yield strength. Equation (5) describes the contribution to strength from particles, $\sigma_{\mathrm{p}}$, with $D_{\mathrm{s}}{ }^{*}$, given as $\sigma_{\mathrm{p}}=B \cdot\left[D_{\mathrm{s}}{ }^{*}\right]^{-1 / 2}$ where $B=395 \mathrm{MPa} \cdot \mu \mathrm{m}^{1 / 2}$. The interparticle spacing $\left[D_{\mathrm{s}}{ }^{*}\right]$ is calculated by knowing the particle diameter $\mathrm{d}$ and the volume fraction of particles $f_{\mathrm{v}}$, eq. (4). For small volume fractions of particle, i.e. 0.01 to 0.02 , the -1 term in eq. (4) is negligible, and $D_{\mathrm{s}}{ }^{*}$ equals $1.08 \cdot f_{\mathrm{v}}^{-0.5} \cdot d$. Substituting this relation into eq. (5) yields:

$$
\sigma_{\mathrm{p}}=A^{*} \cdot f_{\mathrm{v}}^{0.25} \cdot d^{-0.5}
$$

and $A^{*}=379 \mathrm{MPa} \cdot \mu \mathrm{m}^{1 / 2}$. Equation (9) can be modified by normalizing the observed particle size to the smallest conceivable particle size. In an analysis of particle hardening in steels, Onaka ${ }^{38)}$ proposed a smallest particle size of $5 \mathrm{~b}$ where $b$ is the size of an iron atom $(0.287 \mathrm{~nm})$, yielding $d_{\text {min }}=1.4 \mathrm{~nm}$. This is in agreement with Zhao et al. ${ }^{39)}$ who estimate, from thermodynamic arguments, that the minimum embryonic size is about $1 \mathrm{~nm}$. Applying $d_{\min }=1.4 \mathrm{~nm}$ in eq. (9), yields

$$
\sigma_{\mathrm{p}}=A^{* *} \cdot f_{\mathrm{v}}^{0.25} \cdot\left[d / d_{\mathrm{Fe}_{3} \mathrm{O}_{4}}\right]^{-0.5}
$$

where $A^{* *}=10,005 \mathrm{MPa}$. The particle strength, $\sigma_{\mathrm{p}}$, can be normalized to a theoretical strength, $\sigma_{\mathrm{o}}$, making eq. (10) dimensionless. The theoretical strength is related to the 


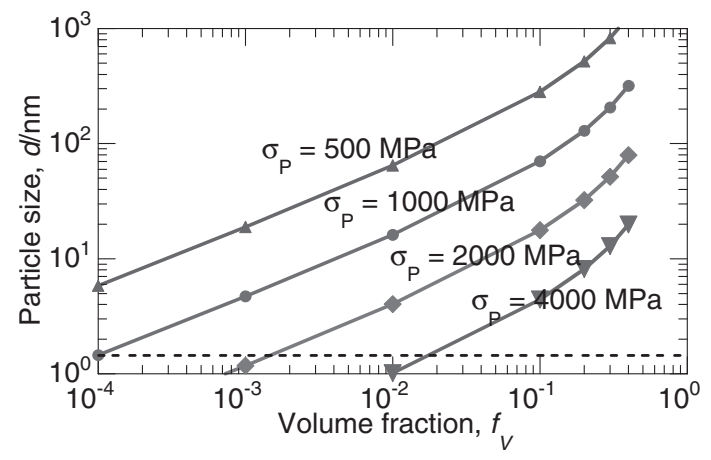

Fig. 8 Predicted strength of ball-milled Fe-O materials from oxide particles plotted as a function of particle size and volume fraction.

modulus $\mathrm{E}$ through a constant $\chi$ given as $\sigma_{\mathrm{o}}=E / \chi$. A calculated value of $\chi=20.7$ was determined to make eq. (10) unit-less. This value is in the order of the theoretical estimate of strength. ${ }^{40)}$ The resulting relation, with $\chi=20.7$ is:

$$
\sigma_{\mathrm{p}} / \sigma_{\mathrm{o}}=f_{\mathrm{v}}^{0.25} \cdot\left[d / d_{\mathrm{Fe}_{3} \mathrm{O}_{4}}\right]^{-0.5}
$$

Figure 8 illustrates the predicted strength from particles in particle-hardened iron (grain and subgrain free) as a function of the particle size and the volume fraction of particles. Isostrength curves are shown at equal increments of increase in strength. The deviation from linearity for the strength change at large volume fractions arises from the use of the rigorous relation eq. (4) for $D_{\mathrm{s}}{ }^{*}$ rather than the approximate one, eq. (9). The graph illustrates that when iron oxide particles are created at the smallest possible size, a volume fraction of $f_{\mathrm{v}}=0.018$ is needed to achieve the strength of $4000 \mathrm{MPa}$. With a ten fold increase in volume fraction, $f_{\mathrm{v}}=0.18$, the particle size will be $7.35 \mathrm{~nm}$ to achieve the same strength. The yield strength, of course, would be higher than $4000 \mathrm{MPa}$ since the contribution from grain size and/or subgrain size must be added to the estimated strength from particles.

It is to be pointed out that eq. (5) is not in agreement with the best known theory proposed for particle hardening where $\sigma_{\mathrm{P}}$ is inversely proportional $d^{-1}$ and not to $d^{-0.5}$. The $d^{-1}$ relation relates to the Orowan bowing stress for motion of dislocations that are pinned by particles spaced $D_{\mathrm{s}}{ }^{*}$ apart. ${ }^{41)}$ Refinement of the Orowan mechanism has been reviewed by Fisher et $a l^{42)}$ Experimental evidence for validity of the bowing stress model is lacking in metallic materials.

\section{Conclusions}

The study of iron-oxygen powders through ball-milling, followed by consolidation, has been pursued by a number of investigators. These studies have revealed remarkable microstructural changes during the processing steps. These include the creation of ultrafine cells, subgrains and grains, as well as particles of iron oxide $\left(\mathrm{Fe}_{3} \mathrm{O}_{4}\right)$ in the nano-size range $(10 \mathrm{~nm})$ adjoining coarser particles. The present investigation is directed towards establishing the influence of oxide particles on the strength of ball-milling and consolidated iron. The following conclusions are presented:
(1) The major contribution to strength arises from the presence of iron oxide particles.

(2) The nano-oxide particles, estimated at $2.5 \mathrm{~nm}$, are created by adiabatic shear banding and phase transformation during ball-milling.

(3) Adiabatically shear banded regions always adjoin work-hardened regions revealing a sharp discontinuity in hardness values.

(4) Ultra-fine grains, although stabilized by the presence of oxide particles, do not contribute significantly to the strength because of grain boundary relaxation.

(5) The Hall-Petch mechanism becomes less effective at grain sizes below $2 \mu \mathrm{m}$.

(6) The strength contribution from particles is given by $\sigma_{\mathrm{p}}=B \cdot\left(D_{\mathrm{s}}{ }^{*}\right)^{-1 / 2}$ where $D_{\mathrm{s}}{ }^{*}$ is the surface to surface interparticle spacing, and $B=395 \mathrm{MPa} \cdot \mu \mathrm{m}^{1 / 2}$.

(7) $D_{\mathrm{s}}{ }^{*}$ for iron-oxygen materials can be calculated from the phenomenological relation $D_{\mathrm{s}}{ }^{*}=0.4 \cdot L$ where $L$ is the grain size, based on the Zener particle pinning concept.

(8) Consolidation by warm rolling of the ball-milled powders leads to growth of the nano-oxide particles to $10 \mathrm{~nm}$.

(9) The consolidated iron containing 8 volume percent of oxide is described by a bi-modal distribution of oxide particles.

(10) Annealing of the consolidated iron-oxygen materials leads to the partial dissolution of nano-oxide particles by grain boundary migration.

\section{Acknowledgements}

This work was performed under the auspices of the U.S. Department of Energy by the University of California, Lawrence Livermore National Laboratory under Contract No. W-7405-Eng-48.

\section{Appendix: Relation of Microstructure to Grain size}

The results shown in Fig. 4 give convincing evidence that nano-size iron oxide particles created during ball-milling is the major source of strengthening iron in the powder and in the consolidated state. The calculation of the interparticle spacing, $D_{\mathrm{s}}{ }^{*}$, from grain size, $L$, was made by a constant $\mathrm{k}$ according to the relation:

$$
k=D_{\mathrm{s}}^{*} / L
$$

The value of $k$ describes the relation of microstructure to grain size. A value of $k=0.4$ was selected based on studies of the fine grain size data of spheroidized UHCS-1.8C material where values of $D_{\mathrm{s}}{ }^{*}$ and $L$ were available. ${ }^{19)}$ Since the study of ball-milled iron and its consolidated state is centered on similar fine grain sizes, it was proposed that this relation is applicable to iron-iron oxide particle materials. Figure A.1 illustrates the values of $k$ as a function of grain size for the UHCS-1.8C material for data from 0.3 to $5 \mu \mathrm{m}$. It is seen that the value of $\mathrm{k}$ is about 0.4 in the ultra-fine grain size range (the average value is 0.44 ) and then decreases abruptly to an average value of about 0.13 in the range above $2.5 \mu \mathrm{m}$. The two regions are marked as I and II respectively. 


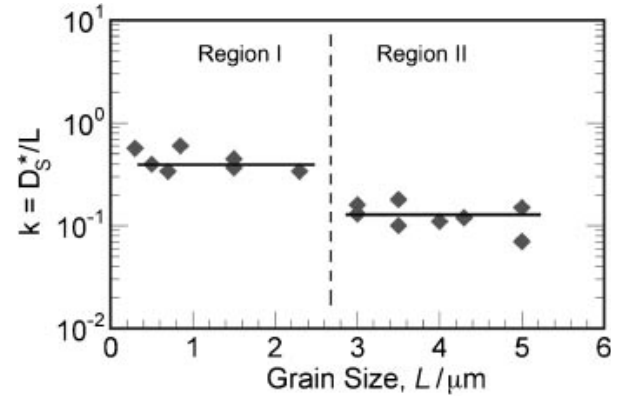

Fig. A.1 Value of $k=D_{\mathrm{s}}{ }^{*} / L$ plotted as a function of the grain size for the UHCS-1.8C material from 0.3 to $5 \mu \mathrm{m}$.

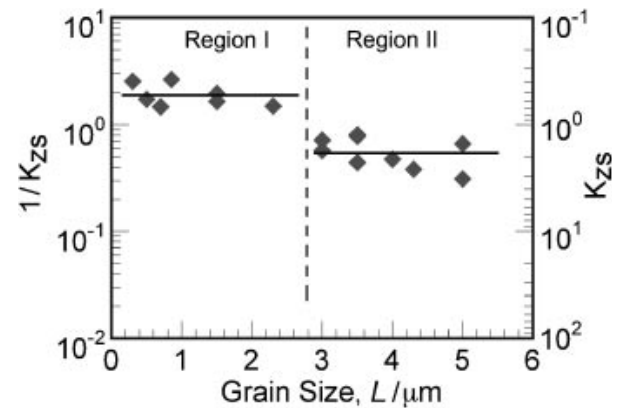

Fig. A.2 The Zener-Smith constant, $K_{\mathrm{zs}}$ (as $\left.1 / K_{\mathrm{zs}}=d \cdot f_{\mathrm{v}} / L\right)$, plotted as a function of the grain size for the UHCS-1.8C material from 0.3 to $5 \mu \mathrm{m}$.

The two distinct regions suggest that the microstructure factors controlling grain size and grain growth have changed. In the case of the UHCS-1.8C material the coarse grain size data (3 to $5 \mu \mathrm{m}$, Region II) was obtained by a DETWAD process. ${ }^{40)}$ This process involves warm working just above the eutectoid temperature followed by phase transformation during continuous working. The high driving force from phase transformation provides sufficient energy to cause rapid grain growth leaving numerous particles within the grains.

The relation $D_{\mathrm{s}}{ }^{*}=0.4 \cdot L$ is phenomenological. It has some common characteristics, however, with the well-known Zener-Smith model for describing grain growth by particle pinning. ${ }^{23)}$ Their model predicts the grain size to be directly proportional to the particle diameter, $\mathrm{d}$, when normalized by the volume fraction, $f_{\mathrm{v}}$, as follows:

$$
L=K_{\mathrm{zs}} \cdot d \cdot f_{\mathrm{v}}^{-1}
$$

The value of $K_{\mathrm{zs}}$ has been predicted theoretically as 1.33. ${ }^{23}$ Equation (A-2) can be written in a similar way as eq. $(\mathrm{A} \cdot 1)$ as follows:

$$
1 / K_{\mathrm{zs}}=d \cdot f_{\mathrm{v}}^{-1} / L
$$

That is, $1 / K_{\mathrm{zss}}$ is equal to the ratio of a microstructure factor to the grain size. Figure A. 2 shows the value of $1 / K_{\mathrm{zs}}$ plotted as a function of the grain size for the same UHCS-1.8C material used earlier for calculating $k$ (Fig. A-1). A similar pattern is evident for $1 / K_{\mathrm{ZS}}$ with two plateau regions, Region I with the average $1 / K_{\mathrm{zs}}$ equal to $1.8\left(K_{\mathrm{zs}}=0.55\right)$ and decreasing in Range II to an average of $0.55\left(K_{\mathrm{zs}}=1.92\right)$. The sudden increase in the $K_{\mathrm{zs}}$ values at grain sizes above
$2.5 \mu \mathrm{m}$ is explained in a similar way as the sudden change for $\mathrm{k}$ discussed earlier. That is, the increase in the size of grains created from phase transformation is larger than the particle size change that was observed in Region I. The average value of $K_{\mathrm{zs}}=0.55$ obtained from the data for UHCS-1.8C lies in the middle of a wide range of values ( 0.15 to 0.9 as documented by Belyakov et al. $).{ }^{11)}$ These values are in contrast to the theoretical value of 1.33 .

Our proposed relation is different from the Zener-Smith equation in that it states that the grain size is uniquely proportional to the surface-to-surface inter-particle spacing, i.e. $L=(1 / k) D_{\mathrm{s}}{ }^{*}$. The relation, however, can be modified to show a close resemblance to the Zener-Smith relation eq. (A.2). This is because the interparticle spacing, $D_{\mathrm{s}}{ }^{*}$, is related to particle size, $d$, and volume fraction, $f_{\mathrm{v}}$, according to the relation:

$$
D_{\mathrm{s}}{ }^{*}=(3 / 2)^{0.5}\left\{\left[\pi /\left(4 \cdot f_{\mathrm{v}}\right)\right]^{0.5}-1\right\} \cdot d
$$

Applying Eq. (A.3) to describe the variation of grain size with $\mathrm{d}$ and $f_{\mathrm{v}}$, one obtains:

$$
L=1 / k \cdot(3 / 2)^{0.5}\left\{\left[\pi /\left(4 \cdot f_{\mathrm{v}}\right)\right]^{0.5}-1\right\} \cdot d
$$

This rigorous relation is simplified when the volume fraction of particles is small (e.g. .03 or less). The term $(3 / 2)^{0.5}$. $\left\{\left[\pi /\left(4 \cdot f_{\mathrm{v}}\right)\right]^{0.5}-1\right\}$ becomes equal to $1.08 \cdot f_{\mathrm{v}}^{-0.5}$. In this case, eq. (A.4) is simplified and given approximately as:

$$
L=1 / k \cdot d \cdot f_{\mathrm{v}}^{-0.5}
$$

In summary, the difference between our proposed relation eq. (A.6) and the Zener-Smith relation eq. (A.2) is in the exponent of the volume fraction term. The volume fraction of particles is a more important variable in the Zener-Smith relation $\left(f_{\mathrm{V}}^{-1}\right)$ than in the proposed relation $\left(f_{\mathrm{v}}^{-0.5}\right)$. The Zener-Smith model, however, relies on a uniform distribution of equal size particles throughout the material. This distribution is rarely observed in real systems. Humphrey and Hatherly ${ }^{43}$ ) have evaluated two different microstructure conditions that were not considered by Zener-Smith. In one example they have shown that when the particle size approaches the grain size (this is the case for the UHCS$1.8 \mathrm{C}$ material) the predicted result agrees with our proposed relation eq. (A.5) with $1 / k=1.6$. In the other example, they show that when all the particles are located in the grain boundary, and are uniformly distributed there, eq. (A.5) is again obeyed with $1 / k=2.8$. These two different theoretical values of $1 / k$ (the average is 2.2) are in close agreement with the experimental value of 2.5 obtained for the UHCS material in Region I.

Convincing experimental evidence for support of the $D_{\mathrm{s}}{ }^{*}$ relation eq. (A.1) for the ball-milled iron studies is from Fig. 4 in the text. Successful correlation of most of the strength data for ball-milled iron in the powder and consolidated states in the range of fine grain sizes was made possible by equating $D_{\mathrm{s}}{ }^{*}$ to $0.4 L$ (that is $k=0.4$ ). Since different oxygen contents were reported by different investigators, it is concluded that the volume fraction of oxide particles is not a variable when $D_{\mathrm{s}}{ }^{*}$ is used as the microstructure variable. This is the situation in Region I where Zener pinning of grain boundaries by particles is the ratecontrolling factor for grain growth. The particle size in the 
grain boundary is known to increase with an increase in grain size. ${ }^{19)}$ In Region II the value of $k$ decreases. This means that more particles will exist within a specific grain. In the case of the UHCS-1.8C material, the change of grain size-particle distribution is related to phase transformation during the DETWAD process (Divorced Eutectoid Transformation With Associated Deformation). ${ }^{44)}$ In the case of the ballmilled iron, the change arises from the rapid grain growth rate that occurs from the high annealing temperature. The result is seen by the separation of the data shown in Fig. 1 of the text above about $2 \mu \mathrm{m}$. The high driving force from heating is sufficient for grain boundary migration to overcome the particle pinning effect. As a result the value of $\mathrm{k}$ is drastically reduced. This is confirmed by values of $\mathrm{k}$ deduced from the coarse grain size materials shown in Figs. 1 and 4. The value of $\mathrm{k}$ is 0.01 for Point $\mathrm{A}(L=10 \mu \mathrm{m})$, and 0.15 for Points B $(2 \mu \mathrm{m})$ and $\mathrm{C}(5 \mu \mathrm{m})$.

\section{REFERENCES}

1) J. C. S. Jang and C. C. Koch: Scripta Metall. Mater. 24 (1990) 1599.

2) Y. Kimura and S. Takaki: Mater. Trans. JIM 36 (1995) 289.

3) Y. Kimura, H. Hidaka and S. Takaki: Mater. Trans. JIM 40 (1999) 1149.

4) J. Yin, M. Umemoto, Z. G. Liu and K. Tsuchiya: ISIJ Inter 41 (2001) 1389.

5) M. Umemoto: Mater. Trans. JIM 44 (2003) 1900.

6) Y. Sakai, M. Otaguchi, Y. Kimura and K. Tsuzaki: Ultrafine Grained Materials, ed. by R. S. Mishra et al., (TMS, Warrendale, PA, 2000), pp. 361-370.

7) A. Belyakov, Y. Sakai, T. Hara, Y. Kimura and K. Tsuzaki: Metall. Mater. Trans. 32A (2001) 1769.

8) A. Belyakov, Y. Sakai, T. Hara, Y. Kimura and K. Tsuzaki: Metall. Mater. Trans. 33A (2002) 3241.

9) A. Belyakov, Y. Sakai, T. Hara, Y. Kimura and K. Tsuzaki: Metall. Mater. Trans. 34A (2003) 131.

10) K. Tsuzaki: Materials Science Forum 426-432 (2003) 2771-2776.

11) A. Belyakov, Y. Sakai, T. Hara, Y. Kimura and K. Tsuzaki: Mater. Trans. 45 (2004) 2252.

12) D. Jia, K. T. Ramesh and E. Ma: Acta Mater. 51 (2003) 3495.

13) S. Takaki, K. Kawasaki and Y. Kimura: J. Materials Processing Technology 117 (2001) 359.

14) R. Armstrong, L. Codd, R. M. Douthwaite and N. J. Petch: Phil. Mag. 7 (1962) 45.

15) N. Tsuji, S. Okuno, Y. Koizumi and Y. Minamino: Mater. Trans. 45 (2004) 2272

16) A. Cracknell and N. J. Petch: Acta Metall. 3 (1955) 186.

17) F. B. Pickering: Physical Metallurgy and the Design of Steels, (Applied Science Publishers, London, 1978), p. 63.

18) D. R. Lesuer, C. K. Syn and O. D. Sherby: Investigations and
Applications of Severe Plastic Deformation, ed. by T. C. Lowe and R. Z. Valiev, 357, (Kluwer Academic Publishers, the Netherlands, 2000), p. 357.

19) C. K. Syn, D. R. Lesuer and O. D. Sherby: Metall. Mater. Trans. 25A (1994) 1481

20) C. K. Syn, D. R. Lesuer and O. D. Sherby: Mater. Sci. Tech. 21 (2005) 317.

21) G. S. Ansell: Physical Metallurgy, ed. by R. W. Cahn, (North Holland Publishing Company, Amsterdam, 1965), p. 887.

22) E. O. Hall: Proc. Roy. Soc. B54 (1951) 747.

23) C. Zener: quoted by C. S. Smith, Trans. Am. Soc. Metals 175 (1948) 15.

24) O. D. Sherby, M. Carsi, W. J. Kim, D. R. Lesuer, O. A. Ruano, C. K. Syn, E. M. Taleff and J. Wadsworth: Mats. Sci. Forum 426-432 (2003) 11.

25) A. Ohmori, S. Torizuka and K. Nagai: ISIJ International 44 (2004) 1063.

26) N. Tsuji, R. Ueji, Y. Minamino and Y. Saito: Scripta Mater. 46 (2002) 305.

27) V. Ramachandran and E. P. Abrahamson II: Scripta Metall. 6 (1972) 282.

28) V. Ramachandran and E. P. Abrahamson II: $4^{\text {th }}$ Int. Conf. on Strength of Metals and Alloys, (ed. by Laboratoire de Physique du SolideENSIMIM INPL, Nancy, France, 1976), p. 1338.

29) Y. Funakawa, T. Shiozaki, K. Tomita, T. Yamamoto and E. Maeda: ISIJ International 44 (2004) 1945.

30) H. Hidaka, T. Tsuchiyama and S. Takaki: Scripta Mater. 41 (2001) 1503.

31) Y. Xu, M. Liu, M. Umemoto and K. Tsuchiya: Metall. Mater. Trans. 33A (2002) 2195.

32) Y. Xu, M. Umemoto and K. Tsuchiya: Mater. Trans. 43 (2002) 2205.

33) D. R. Lesuer, C. K. Syn and O. D. Sherby: Mater. Sci. Eng. (2006) submitted

34) C. M. Young and O. D. Sherby: J. Iron Steel Inst. 211 (1973) 640.

35) D. R. Lesuer, C. K. Syn and O. D. Sherby: Mater. Sci. Eng. 410-411 (2005) 222

36) D. R. Lesuer, C. K. Syn and O. D. Sherby: Proceedings MS\&T 2004 Conference, New Orleans, LA, October 2004, (TMS, Warrendale, PA, 2004), pp. 123-133.

37) M. Hansen and K. Anderko: Constitution of Binary Alloys, (McGraw Hill Book Company, New York, 1958), p. 685.

38) S. Onaka: quoted as private communication in reference (11).

39) M. C. Zhao, T. Hanamura, H. Qui and K. Yang: Mater. Trans. 46 (2005) 784.

40) A. H. Cottrell: Dislocations and Plastic Flow in Crystals, (Oxford at the Clarendon Press, 1953).

41) E. Orowan: Symposium on Internal Stresses, (Institute of Metals, London, 1948), p. 451

42) J. C. Fisher, E. W. Hart and R. H. Pry: Acta Met. 1 (1953) 336.

43) F. J. Humphreys and M. Hatherly: Recrystallization and Related Phenomena, (Pergamon Press, Oxford, 1996), pp. 307-309.

44) T. Oyama, J. Wadsworth, B. Walser and O. D. Sherby: Scripta Met. 18 (1984) 799 . 